\title{
Self-events and other-events: Temporal dating and event memory
}

\author{
ANDREW L. BETZ \\ GTE Laboratories, Waltham, Massachusetts \\ and \\ JOHN J. SKOWRONSKI \\ Ohio State University, Newark, Ohio
}

\begin{abstract}
A diary methodology was used to assess factors related to temporal dating and cued recall of realworld events. In one diary, participants kept a record of unique personal autobiographical events. In a second diary, participants recorded unique events from the life of a friend or relation. At the time each event was recorded, participants rated the event's pleasantness, person typicality, and degree of initial mental involvement in the event. At the end of the academic quarter, participants provided a recall rating, a rehearsal rating, a date estimate, and a report of the strategy used to estimate the date for each event. Results of regression analyses indicated that both self-events and other-events were characterized by superior memory for person-atypical events. Furthermore, there was a positivity bias in recall for self-events, but there was a negativity bias in recall for other-events. Mediational analyses indicated that the self-event positivity bias was due to enhanced mental involvement when the events occurred, whereas the other-event negativity bias was due to subsequent event rehearsal. The date estimation results indicated that self-event dating was more accurate and evinced less telescoping than other-event dating. Furthermore, the accuracy of date estimates was substantially mediated by event memory. However, mediational differences between self-events and other-events did not emerge. The theoretical implications of these results are discussed.
\end{abstract}

Various aspects of memory for real-world events have recently received substantial empirical attention (see, e.g., G. Cohen, 1989; Conway, 1990). Two of the more active areas include memory for the content of real-world events (see, e.g., Robinson, 1992; Rubin, 1982, 1996) and memory for the dates on which events occurred (Friedman, 1993; Thompson, Skowronski, Larsen, \& Betz, 1996). Because event dates are often reconstructed rather than recalled, and because the content of event memory is one of the prime sources of material for this reconstruction, our own research often has simultaneously collected information on both event memory and event dating (Skowronski, Betz, Thompson, \& Larsen, 1995; Skowronski, Betz, Thompson, \& Shannon, 1991; Thompson, Skowronski, \& Betz, 1993).

In one of our prior studies, we compared event memory and event dating for two types of autobiographical events: self-events and non-self events (Skowronski et al., 1991). The non-self events were the events of close friends or relatives of the study participants. Although previous

We thank the students from The Ohio State University at Newark who helped to conduct the diary test sessions and to content-code the diary entries: Laura Shannon, Jeannie Rausch, Michelle Monroe, Mary McGovern, Robin Butler, and Julie Hott. We also thank Purdue University for facilities provided to the second author during the revision of this manuscript. Correspondence should be addressed to A. L. Betz, GTE Laboratories, Inc., 40 Sylvan Road, Waltham, MA 02254 (e-mail: abetz@gte.com). studies have investigated autobiographical memory for some types of non-self events, such as public events (Larsen, 1988; Larsen \& Thompson, 1995), to date there have been no additional studies explicitly comparing event memory and event dating for self-events and the events of close friends and relations. Hence, in the present paper, we sought to replicate, extend, and further explore the findings of our initial study. In the following sections, we highlight some of the theoretical issues in memory and event dating that guide this research, review the findings of our initial study, and explain how the present research potentially can extend the results of our earlier study.

\section{TEMPORAL JUDGMENT AND SOURCES OF TEMPORAL INFORMATION}

In a series of recent papers, we examined how people judge when autobiographical events occurred (e.g., Skowronski et al., 1995; Thompson et al., 1993; also see Thompson et al., 1996). Our data revealed that direct retrieval of the actual date of an event from memory does not often occur. Instead, people usually reconstruct event dates, using many sources of information to do so (see also, Brewer, 1988; N. R. Brown, 1990). As one might reasonably expect, when the accuracy of these sources of information decreases, the corresponding accuracy of date reconstructions also decreases. However, merely showing that people reconstruct event dates does not tell 
the whole story. Our past research has also paid special attention to three reconstruction strategies that are used in event dating.

First, a known (or supposedly known) event date can serve as an anchor, and the dates of other events are judged relative to that anchor. Such event relation strategies encompass both dates estimated relative to a single anchor event (e.g., "That happened a week after my birthday.") and events that must logically maintain a chronological order (e.g. "I got into a fender bender, then the next day took my car to the shop."). These relational strategies are probably also responsible for bounding effects in event dating, including both telescoping and time expansion, in that the bounding dates serve as anchors (Huttenlocher, Hedges, \& Bradburn, 1990; Skowronski et al., 1995; Thompson, Skowronski, \& Lee, 1988).

A second reconstruction strategy uses memory for event content to produce the event date (e.g., "The event happened when the leaves on the trees were turning color, so it must have happened in the fall."). Presumably, as one recalls a greater number of event details, the accuracy of the date estimate will increase, especially if the event details provide temporal information. A proxy measure of memory for event details is self-reported goodness-of-memory. Dating accuracy is positively related to self-reported goodness-of-memory, but, sensibly, this reconstruction strategy produces estimates that are less accurate than is direct recall of event dates (Thompson et al., 1993).

A third strategy relies on people's real-world knowledge structures. For example, one might know that people generally go to church on Sunday. Hence, when dating an event that happened in church, people might logically infer that the event was on a Sunday. One likely consequence of this strategy is that date estimates should often be assigned the wrong date, but the correct day of the week. Previous research has demonstrated exactly this pattern (Skowronski et al., 1991; also see Larsen \& Thompson, 1995).

Although our initial study comparing dating of selfevents versus dating of other-events revealed that selfevent dating was more accurate, that study provided incomplete evidence concerning the mental processes or strategies that might underlie this difference. Event memory was better for self-events than for other-events, and thus, it is possible that the more accurate dating of selfevents could be attributed to differences in event recall. However, no data were collected with respect to the other estimation strategies that people use to reconstruct event dates. Hence, it is not known whether these other strategies also play a role in explaining the self versus other dating accuracy differences that we obtained. In the present study, we again examined the patterns of self-event dating and other-event dating, but paid special attention both to the strategies used to date events and how those strategies might differentially affect the dates assigned to self-events and other-events.

\section{STIMULUS CHARACTERISTICS, MEMORY FOR SELF-EVENTS, AND MEMORY FOR OTHER-EVENTS}

In addition to its use in explaining accuracy in event dating, memory for real-world events is obviously interesting in its own right. In previous papers, we have extensively explored some of the stimulus characteristics that are associated with the recall of real-world selfevents. However, as with event dating, few of these characteristics have been explored with respect to recall for other-events. With the exception of the Skowronski et al. (1991) research, the empirical focus has been almost entirely on self-events.

Thus, one major purpose of the present paper was to extend the research by Skowronski et al. (1991) and further explore whether some of the event characteristics that are related to memory for self-events are also related to memory for other-events. In the present paper, we focus on how event valence and event person typicality are related to recall of both self-events and of otherevents. In addition, we attempt to gain insight into some of the cognitive mechanisms that may be responsible for these effects.

\section{Event Valence}

The usual finding with respect to event valence, obtained in both laboratory and real-world contexts, indicates that pleasant self-events are recalled more quickly and are remembered better than are negative self-events (e.g., Matlin \& Stang, 1978; Skowronski et al., 1991; Wagenaar, 1986). Surprisingly, the cognitive mechanisms underlying this positivity effect remain unspecified.

One possible mechanism is selective rehearsal (see, e.g., Matlin \& Stang, 1978). Pleasant events may be rehearsed more frequently than unpleasant events, and it may be this rehearsal that is responsible for the heightened memory accessibility and memory clarity for positive events. This selective rehearsal need not be intentional. For example, Freud (1957/1915) suggested that negative, potentially threatening material may be unconsciously repressed. However, regardless of whether the rehearsal is intentional or unconscious, the end result is the same: better memory for positive self-events than for negative self-events.

Despite the intuitive appeal of this selective rehearsal mechanism, there are reasons to suspect that it is not solely responsible for positivity effects in recall. The flashbulb memory literature indicates that although rehearsal is sometimes implicated in the relation between event vividness and memory, rehearsal is not a necessary condition for existence of a vividness-memory relation (R. Brown \& Kulik, 1977; Pillemer, 1984; Rubin \& Kozin, 1984; Winograd \& Killinger, 1983). For example, in one study of memories that were 2 to 22 years old, elevated emotional reactions were associated with accessible mem- 
ories, but not rehearsal (Pillemer, Goldsmith, Panter, \& White, 1988).

A second reason to doubt the sufficiency of the selective rehearsal mechanism is that a positivity finding is not inevitable. For example, Kreitler and Kreitler (1968) obtained a negativity effect in autobiographical memory. This outcome suggests that conditions exist in which positivity effects in memory can be eliminated or even reversed. One such condition may be whether the events recalled primarily pertain to the self or another person. Supporting this suggestion, in our earlier study we found a positivity effect in recall for self-events, but not for other-events (Skowronski et al., 1991).

A second possible explanation for the positivity bias in event memory is the notion that positive events are more accurately processed at encoding than are negative events (see Matlin \& Stang, 1978). That is, positive events may be "savored" as they occur, with people being mentally open and active during the event. This enhanced processing should facilitate later recall of event details. By comparison, negative events may cause a shutdown of processing. In particular, during negative events people may try to minimize negative affect by ignoring details as the event transpires (but see Taylor, 1991). For example, in an attempt to minimize the pain, some people avert their eyes as the inoculation needle approaches their arm.

In the present paper, in order to explore their possible roles in producing valence biases in recall, we assessed both the amount of initial processing and the amount of rehearsal given to each event. We then examined whether these factors mediated valence biases in recall.

\section{Event Person Typicality}

A second characteristic of interest in the present paper concerns the impact of trait expectancies on event recall. Some laboratory research shows that events inconsistent with a prior trait expectancy for a person yield the best recall, events consistent with a prior trait expectancy yield middling recall, and events irrelevant to a trait expectancy yield the worst recall (for a review, see Stangor \& McMillan, 1992). Despite others' claims that the laboratory findings were mere artifacts of highly artificial stimuli or restricted stimulus presentation time (e.g., Neisser, 1982), we found this exact pattern in our initial autobiographical memory study comparing self-event recall and other-event recall. Moreover, our prior study revealed this pattern for both self-events and otherevents-a new finding.

The generally accepted explanation for this recall pattern focuses on an individual's mental activity during an event. According to this explanation, events inconsistent with a trait expectancy invoke a process of reconciliation as the individual tries to square the surprising event with the expectancy. This enhanced processing may affect both the strength of the memory trace for the incongruent item (see, e.g., Hastie, 1984) and the connections between the item and other person-relevant information (see, e.g., Srull, Lichtenstein, \& Rothbart, 1985).
The enhanced processing resulting from reconciliation may leave different markers in the data. On the one hand, reconciliation processes imply that people are more mentally involved when an event happens and expend extra effort thinking about these events. If so, assessing degree of initial mental involvement might help demonstrate the underpinnings of the person-incongruity findings in a real-world context. On the other hand, thinking about person-inconsistent information may also occur some period of time after the event occurs. Perhaps individuals expend some effort when an inconsistent event occurs, only to decide that further reflection is needed later. Were this true, a relation between event rehearsal and person incongruity might occur. Thus, the same initial processing and rehearsal measures that were included to explore possible causes of valence effects in recall might also be useful in understanding incongruency effects.

To summarize, in addition to replicating the patterns of data obtained in Skowronski et al. (1991), we seek in this paper to advance our previous research in two important ways. First, we attempt to investigate some of the cognitive mechanisms underlying the memory effects obtained by Skowronski et al. (1991) and also explore whether those mechanisms are different for self-event recall versus other-event recall. Second, we investigate possible differences in self-event and other-event dating accuracy, as well as whether the strategies people use to reconstruct event dates differ for self-events and otherevents.

\section{METHOD}

\section{Participants}

All 49 participants were recruited from undergraduate psychology courses at The Ohio State University at Newark, and they received course credit as compensation for their participation.

\section{Event Recording and Preratings}

Participants kept two diaries, one for self-events and one for the events of another person. Participants chose this person themselves and were instructed to choose someone they saw daily. Most of these others were roommates, close friends, or family members.

Each day, participants recorded two events, one in their self-diary and one in their other-diary. The recording spaces in the diaries were already dated, and participants simply wrote a description of the event in the correct space on each diary page. The events recorded were subject to several constraints. First, events should be unique. The definition of unique supplied to participants was that an event should happen only once in an academic term and should have characteristics that distinguished it from potentially similar events. Second, when a variety of events was available, events should be chosen so that they spanned a wide range of pleasantness, typicality, and involvement. Third, each event should be described in no more than three lines of text. Finally, as much unique information about each event (within the constraints) should be provided. To encourage such behavior, participants were told that their memory for the events would be assessed later, and that their performance in the diary test session would be optimal if their event descriptions were maximally informative.

At the time of recording, participants also provided several ratings for each event. The first of these was a person-typicality rating. Participants were asked to rate each event in terms of how typ- 
ical it was for the person involved in the event. It was emphasized that this rating was person specific. For example, participants were told that getting a $\mathrm{C}$ on a psychology exam could be very typical if the diary target usually got $\mathrm{Cs}$, but could be very atypical if the diary target usually got As. Participants made this rating on a $7-$ point scale ranging from -3 (very atypical) to +3 (very typical). The second rating assessed participants' perceptions of event pleasantness. This rating was made on a 7 -point scale, where -3 was very unpleasant and +3 was very pleasant. The third rating assessed perceptions of the level of mental involvement experienced with each event. Specifically, participants were told that there may be some events in which they are very mentally "into" the event as it happens (e.g., a concert) and other events in which their level of mental activity is quite low (e.g., a psychology lecture). Participants were asked to rate the level of mental involvement they experienced with each event as it was happening on a 5-point scale, where 1 was not at all involved and 5 was extremely involved.

\section{Procedure}

Event recording. Participants recorded events for roughly the length of the 10-week academic term, turning in their diary sheets weekly. Participants entered events into their diaries until the day before their testing sessions. Because of practical constraints on testing sessions (e.g., interviewer availability), not all individuals were tested on the same day. As a result, the exact length of diaries varied slightly across individuals. The age of the oldest diary event ranged from a low of 69 days old to a high of 73 days old.

Testing event memory and event dating. The testing sessions were conducted during the final exam week of the academic term. As a counterbalancing factor, half the participants were randomly assigned to be tested over their self-diary first, and the other half were tested over their other-diary first

All tests were conducted individually. The testing was conducted by reading aloud each person's diary events, one at a time, in random order. After hearing each event, participants first decided whether the event was unique. If it was not, the event was simply discarded. This was done to minimize the possibility of contaminating the dating estimates due to multiple, nondiscriminable occurrences of the same event. After deciding whether an event was unique, participants rated the event's memorability. The scale used was taken from Herrmann and Neisser (1978): (1) not at all, (2) barely at all, (3) not so well, (4) fairly well, (5) very well, (6) almost perfectly, and (7) perfectly. Participants were explicitly advised that a rating of 7 meant perfect memory-for example, "If the event involved a conversation, you could recall the conversation word for word."

After reporting their memory rating, participants provided a rehearsal rating for the event. Specifically, participants reported how often they thought about the event after it occurred. The scale used in this judgment was as follows: (1) not at all, (2) once or twice, (3) several times, (4) quite often, and (5) every day or nearly every day.

Participants then attempted to date the event. They did so by referring to a 4-month calendar containing month names, dates, and day-of-week names. A block of days on the calendar was sequentially numbered from 1 to 110 , with the first number in the sequence indicating a date about 2 weeks prior to the start of the academic term. This was done to obscure the exact day on which the academic term began. These sequential numbers were used to make date reporting more convenient. Participants indicated their date estimate by calling out the number corresponding to the date on which they believed the event occurred.

After reporting a date estimate, participants were asked to indicate the information sources that they had used to remember or reconstruct the date estimate. The sources provided were as follows: (1) knowing the exact date, (2) relating the event to another event that served as a dating reference point, (3) knowing the general period in which the event occurred, (4) estimating the number of events that have happened in the intervening retention interval, (5) using the clarity of memory to estimate the event's date (e.g., clear $=$ recent, poor $=$ old $),(6)$ using a prototypic date (e.g., if this was a class it had to be on a Monday), (7) a guess, or (8) another source not listed (data using these information types are also reported in Thompson et al., 1988; Thompson et al., 1993; also see Linton, 1975).

After all events had been rated and dated, participants were debriefed and dismissed.

$A$ brief word about the memory ratings. Our memory measure is, in effect, a cued-recall meta-memory measure. It is cued recall in the sense that the diary entries, when read aloud, prompt recall for the event--and participants are clearly told they are to rate memory for the event, not for the description. Second, it is a metamemory measure because it involves the self-assessment of the "goodness" of a memory, rather than involving a direct assessment of memory itself. The use of meta-memory measures in studies such as these is discussed at length elsewhere (see Rubin, 1982; Skowronski et al., 1991; Thompson, 1982, 1985; Thompson et al., 1996). However, several points are worth emphasizing.

First, although it is true that the self-report measure of memory might contain biases, the same can be said for other memory measures. Recognition measures are subject to guessing biases, freerecall measures can be insensitive to some information (e.g., implicit memory), and free recall may not provide a thorough assessment of the contents of recall (as illustrated by the phenomenon of hypermnesia). We seek not to belittle these traditional measures, but to point out that there is no perfect way to assess memory. One implication of this point is that to control for the biases contained in each measure, multiple measures of memory are in order. Importantly, toward this end, evidence now suggests that the meta-memory measures used in this study are strongly correlated with other, more traditional measures of memory (see, e.g., Burt \& Kemp, 1991; Leonesio \& Nelson, 1990). Furthermore, the positivity and extremity effects for self-event memory reported in Skowronski et al. (1991) have appeared in other autobiographical memory studies using different recall assessment techniques (e.g., Brewer, 1988; Linton, 1975; Wagenaar, 1986). Hence, although our meta-memory measure may not be perfect, convergent validity data strongly support its utility in assessing memory (see Herrmann, 1984).

A second concern that occasionally arises is that participants memory ratings are epiphenomenal, reflecting their theories about memory rather than their actual recall. Given the methodology used in these studies, such an argument is far-fetched. First, in the methodology that we use, participants do not see their original ratings. Hence, in order to use their theories to concoct the memory data, they would have to first retrieve their original ratings. Second, such an explanation must assume that participants have relatively sophisticated theories of memory. For example, to construct the data from Skowronski et al. (1991), participants would need to know that both expectancy-incongruent and expectancy-congruent information show enhanced recall and that the impact of event valence on self-memory differs from the impact of event valence on other-memory. Third, and most importantly, there are a large number of factors under investigation in these studies. For example, in the present study, for participants to use their theories about memory to gerrymander the data, they must report their memory ratings so that they simultaneously satisfy the constraints of at least eight factors that may affect memory (e.g., linear event age, quadratic event age, extremity, valence, rehearsal, mental involvement, person typicality, person atypicality), and must do so on-line. From the perspective of both cognitive capacity and the principle of least effort, this does not seem likely.

Our observations of the participants' behavior in the diary testing sessions are, instead, consistent with the idea that participants directly and honestly report their perceptions of their own memory, and they do so without extensive expenditure of time and effort. Despite all the ratings, rankings, and date estimations that are required during the test, most participants finish their testing session in 
about an hour. Given that during the diary interview session participants are making five judgments about many events (typically from 100 to 120), and that the event dating judgment is typically the task that consumes the majority of the time, such a high rate of speed is inconsistent with a high level of considered hypothesis test ing for the memory judgments. It is far more likely that participants are doing just what we ask: accessing the contents of memory to make their meta-memory ratings.

\section{RESULTS}

\section{Our Approach to Data Analyses}

We used pooled within-subject multiple linear regression for inferential analyses of our data. A benefit of this approach is that the effect tests are more powerful than effect tests conducted in an analysis of variance (ANOVA) framework. Specifically, the ANOVA framework would have required that, to avoid missing values, we collapse across observations within subject. In contrast, the regression framework allows missing values, thus facilitating the use of multiple observations from each participant in the analyses.

However, there are drawbacks with such an approach. First, many regression models could be used. We selected models on the basis of methodological considerations and a priori theoretical interests. Our analytic approach is a derivative of the hierarchical regression technique discussed in J. Cohen and P. Cohen (1983). In simplest terms, we conducted a series of regressions of increasing complexity, interpreting only the highest order effects contained in each analysis. For example, in an analytic series, our initial model would include only the main effects of interest. A second model would include all two-way interactions, as well as the main effects, but only the two-way interactions would be treated as meaningful. Analysis of higher order interactions would proceed in a similar fashion.

To help control for nonindependence of observations, participants were assigned dummy codes and treated as categorical variables in all analyses. However, although this technique controls for nonindependence due to subjects, it does not control for nonindependence due to measures. To the extent that such nonindependence exists, error terms in the analyses will be understated and degrees of freedom will be overstated. To compensate for this difficulty, we adopted a conservative significance criterion of .01 for all analyses.

Both recall and absolute dating error may vary both linearly and quadratically with event age. Thus, we included terms for the linear effect of event age and the quadratic effect of event age as covariates in all regression models. As a consequence, all statistically reliable effects that we report control for both the linear age and quadratic age of events.

Research suggests that the emotional tone of an event can be abstracted into two orthogonal components: valence and extremity (Diener, Larsen, Levine, \& Emmons, 1985; Reisberg, Heuer, McLean, \& O'Shaughnessy, 1988; Thomas \& Diener, 1990). Accordingly, we derived an extremity measure by taking the absolute value of the participants' event pleasantness ratings for each event, a procedure we have used previously (Skowronski et al., 1991). ${ }^{1}$ Both extremity and the affective direction of the event (which we shall subsequently refer to as "valence") are used as predictors in all regressions that we report. Thus, any conclusions about event valence control for event extremity, and vice versa.

Because the memory ratings will be one of the primary covariates in our analyses of event dating, we first will present the results for our memory measure. Then, in subsequent sections, we will address the data on dating accuracy and error.

\section{Memory for Events}

Event valence, event extremity, diary target, and person typicality. In one set of analyses, we sought to determine whether event memory would be related to event valence, person typicality, event extremity, and diary target (self-event or other-event). On the basis of both laboratory research and the results of our earlier self-event versus other-event study, we expected events that were more extreme to be better recalled than events that were less extreme. This expectation was confirmed $[F(1,4307)=125.27, p<.001, \beta=.159]$. We expected self-events to be better recalled than other-events. This expectation, too, was confirmed [self $M=4.41$; other $\left.M=3.83),{ }^{2} F(1,4307)=115.00, p<.001\right]$.

Although there was no overall recall advantage for positive events over negative events $[F(1,4307)=.10, p=$ $.76, \beta=-.004]$, the event valence $\times$ diary target interaction was significant $[F(1,4301)=13.51, p<.001]$. To investigate this interaction, we conducted separate regressions on the self-recall data and the other-recall data. Although it did not meet our stringent .01 significance criterion, for the self-events there was some tendency for positive events to be better recalled than negative events $[F(1,2285)=4.74, p=.030, \beta=.040]$. However, for other-events, negative events were reliably recalled better than positive events $[F(1,1970)=10.59, p=.001$, $\beta=-.066$ ]. In our earlier self-other study (Skowronski et al., 1991), we also obtained a reliable event valence $X$ diary target interaction. In that study, we obtained a significant positivity effect for recall of self-events, but no significant valence effect for other-events. Thus, across both of these studies, positivity does not appear to apply to the recall of others' events, and, in fact, recall of these other-events may be characterized by negativity.

The results of our analyses of the person-typicality variable were also quite sensible. First, given both laboratory studies and the results of our earlier self-other study, we expected person-atypical events to be better remembered than person-typical events. This expectation was confirmed $[F(1,4307)=53.24, p<.001, \beta=$ $-.110]$. However, laboratory research indicates that both expectancy-congruent and expectancy-incongruent events are better remembered than expectancy-neutral events. Accordingly, we expected both high person typicality and high person atypicality to lead to enhanced recall. 
Table 1

Rated Recall (Raw Means) by Level of Person Typicality Person Typicality Rating

\begin{tabular}{|c|c|c|c|c|c|c|}
\hline \multicolumn{7}{|c|}{ Person Typicality Rating } \\
\hline-3 & -2 & -1 & 0 & 1 & 2 & 3 \\
\hline 4.48 & 4.19 & 4.01 & 4.07 & 3.91 & 3.91 & 4.06 \\
\hline
\end{tabular}

As the raw means in Table 1 indicate, this did not occur. Only the person-atypical events evinced enhanced recall.

The analyses of the recall data also yielded an unanticipated interaction among event valence, event extremity, and event person typicality $[F(1,4297)=10.92$, $p<.001]$. To clarify this interaction, separate follow-up regression analyses investigating the effect of event typicality at each combination of event valence and event extremity were performed. The typicality $\beta$ s are presented in Table 2. These data indicate that the recall advantage of person-atypical over person-typical events was particularly strong for events of neutral valence and for events of low and moderate positivity. This pattern suggests that event person typicality may be related to recall only when other factors (e.g., extremity, valence) are not operating to affect recall. ${ }^{3}$

Is event valence related to event memory via rehearsal? In additional analyses, we addressed whether the relation between event valence and recall is mediated by differential rehearsal. Although an overall positivityrehearsal outcome is not supported by the negativity effect that we obtained in recall for other-events, the positivity bias for self-events and the negativity bias for other-events could still possibly be mediated by differential rehearsal. This would require that positive selfevents be more frequently rehearsed than negative selfevents, but that negative other-events be more frequently rehearsed than positive other-events. If this pattern were obtained, and if valence biases in event memory are mediated by rehearsal, then inclusion of the rehearsal index in the regression models should eliminate the relation between event valence and event recall.

Analyses of rehearsal as a dependent measure. Before exploring rehearsal's mediation of the valencememory relation, we assessed the relations between our four main predictors (event extremity, event valence, event person typicality, diary target) and rehearsal. Two outcomes suggest that rehearsal can potentially account only for negativity effects in the recall of other-events. First, contrary to those who claim that people think about positive events more often than negative events, in these data people reported that they rehearsed negative events more frequently than positive events $[F(1,4306)=$ $21.54, p<.001, \beta=-.066]$. Furthermore, there was no event valence $\times$ diary target interaction $[F(1,4300)=$ $.03, p=.873]$, showing that people rehearsed negative events in general, regardless of whether the events pertained to self or other.

Other outcomes of the rehearsal analyses were straightforward. Self-events $(M=2.11)$ were rehearsed more frequently than other-events $[M=1.73, F(1,4306)=$
$55.10, p<.001]$. Rehearsal was marginally more frequent for person-atypical events than for person-typical events $[F(1,4306)=5.97, p=.015, \beta=-.039]$. Rehearsal was also more frequent as event extremity increased $[F(1,4306)=114.84, p<.001, \beta=.162]$. However, event extremity interacted with diary target $[F(1,4300)$ $=8.42, p<.01]$. Separate analyses for self-events and other-events show that reported rehearsal was more strongly related to event extremity for self-events than it was for other-events, although in both cases the relationship was clearly significant $[F(1,2284)=80.60, p<$ $.001, \beta=.183$ for self-events; $F(1,1970)=39.24, p<$ $.001, \beta=.140$ for other-events].

Analyses of memory including the rehearsal covariate. The results exploring the relation between event valence and rehearsal suggest that rehearsal cannot be mediating the positivity effect in self-recall, but could be a mediator of the negativity effect in other-event recall. The covariate analyses looking at the relation between event valence and event memory bear this out. As event rehearsal increased, event memory increased as well $[F(1,2283)=202.93, p<.001, \beta=.259]$. Furthermore, the valence effect in self-event memory, which only approached significance in our original analysis, was also reliable $[F(1,2283)=11.00, p<.001, \beta=.058]$. This outcome suggests two conclusions. First, there is a positivity effect in self-event memory. Second, it is unlikely that event rehearsal is a mediator of this effect.

However, analyses of the other-events show where rehearsal may exert its influence. For other-events, the rehearsal covariate was again significant $[F(1,1969)=$ $693.42, p<.001, \beta=.496$ ], sensibly showing that event memory was positively related to event rehearsal. Furthermore, the rehearsal covariate eliminated the negativity effect in recall for other-events $[F(1,1969)=2.73$, $p=.099, \beta=-.029]$. Thus, these data indicate that people may have preferential recall for the negative events of others because they rehearse those events more frequently than they rehearse positive events.

We also conducted exploratory analyses examining whether rehearsal mediates any of the other relations between our four main predictors and event memory. In these new mediational analyses, the covariate was again significant: Increases in event rehearsal were reliably related to increases in event memory $[F(1,4305)=803.74, p<.001$, $\beta=.375]$. However, despite the presence of this covariate, the effects obtained in our initial analyses remained [event valence $\times$ diary target interaction, $F(1,4299)=$ $15.52, p<.001$; event extremity, $F(1,4305)=56.02$, $p<.001, \beta=.099$; diary target, $F(1,4305)=70.41, p<$

Table 2

Typicality $\beta$ s for Recall $\times$ Event Extremity and Event Valence

\begin{tabular}{lcccc}
\hline \multirow{2}{*}{$\begin{array}{c}\text { Event } \\
\text { Valence }\end{array}$} & \multicolumn{4}{c}{ Event Extremity } \\
\cline { 2 - 5 } & -.171 & 1 & 2 & 3 \\
\hline Negative & - & -.002 & -.021 & -.078 \\
Positive & - & -.202 & -.154 & -.072 \\
\hline
\end{tabular}


.001 ; person typicality, $F(1,4305)=47.97, p<.001$, $\beta=-.096$; event valence $\times$ event extremity $\times$ person typicality, $F(1,4295)=10.30, p=.001]$. Hence, rehearsal is not the sole mediator of any of these effects.

Is event valence related to event memory via initial mental involvement? In another set of analyses, we addressed the possibility that the valence-recall relation is mediated by differential initial mental involvement in the events. As with rehearsal, the negativity bias in recall for other-events indicates that the involvement mechanism must be modified to account for the impact of event valence on memory. That is, for involvement to account for both positivity in self-event recall and negativity in other-event recall, participants would have to report higher mental involvement in positive self-events than negative self-events, but higher mental involvement in negative other-events than positive other-events. If this pattern were obtained, and if valence biases in event memory are mediated by initial mental involvement, then inclusion of the involvement variable in the regression models should eliminate the relation between event valence and event recall.

Analyses of mental involvement as a dependent measure. As with rehearsal, we first conducted a series of analyses in which we explored the potential relations between our four main predictors and mental involvement. The results of this analysis were congruent with the hypothesis that initial mental involvement mediates selfpositivity in event recall but not other-negativity in event recall. Overall, positive events engendered more mental involvement than negative events $[F(1,4265)=77.83$, $p<.001, \beta=.117]$. Extreme events were associated with more mental involvement than moderate events $[F(1,4265)$ $=424.54, p<.001, \beta=.280]$, and a significant event extremity $\times$ diary target interaction $[F(1,4259)=9.79$, $p<.01]$ indicated that this extremity effect was slightly stronger for self-events $[F(1,2263)=424.54, p<.001$, $\beta=.301]$ than for other-events $[F(1,1950)=185.90$, $p<.001, \beta=.276]$. Furthermore, self-events engendered greater mental involvement $(M=3.97)$ than otherevents $[M=2.96, F(1,4265)=520.12, p<.001]$.

Two other unexpected outcomes emerged from this analysis. The first of these was a significant person typicality $\times$ diary target interaction $[F(1,4259)=6.72, p<$ $.01]$. Separate analyses conducted on the self-events and other-events indicated a slight tendency for typical selfevents to be rated as more mentally involving than atypical self-events $[F(1,2263)=7.43, p<.01, \beta=.057]$. There was no such effect for other-events $[F(1,1950)=$ $.06, p=.80, \beta=-.005]$.

Analyses of memory including the initial mental involvement covariate. The previous analyses suggest that mental involvement may mediate the positivity bias in self-event recall, but not the negativity bias in otherevent recall. Recall analyses including mental involvement as a covariate support this idea. The involvement covariate was significant for self-events $[F(1,2262)=$ $52.95, p<.001, \beta=.145$ ], suggesting that events producing high mental involvement were also well remembered. Further, as would be expected if mental involvement mediated the positivity bias for self-events, inclusion of the involvement covariate in the regression model rendered the event valence term nonsignificant $[F(1,2262)=1.15, p=.28, \beta=.019]$. Hence, initial mental involvement is a plausible mediator of the positivity effect in self-event recall. However, this conclusion must be drawn with caution, for the valence effect was only marginally significant in the original analysis.

By comparison, the mental involvement covariate was also significant in the analysis of other-events $[F(1,1949)$ $=19.10, p<.001, \beta=.104]$. However, the involvement term failed to eliminate the negativity bias in other-event recall $[F(1,1949)=15.22, p<.001, \beta=-.082]$. Hence, initial mental involvement is not a plausible mediator of the negativity effect in other-event recall.

We also conducted exploratory analyses examining whether initial mental involvement mediates any of the relations between our additional predictors and event memory. In these new mediational analyses, the covariate was again significant: Increases in initial mental involvement were reliably related to increases in event memory $[F(1,4264)=46.04, p<.001, \beta=.106]$. Despite this significant covariate, the effects that we obtained in our initial analyses were maintained [event valence $\times$ diary target interaction, $F(1,4258)=8.30, p<$ .01 ; event extremity, $F(1,4264)=76.27, p<.001, \beta=$ .127 ; diary target, $F(1,4264)=62.37, p<.001$; person typicality, $F(1,4264)=57.39, p<.001, \beta=-.113$ ]. Thus, none of these effects are solely mediated by initial mental involvement. ${ }^{4}$

\section{Event Dating}

A primary goal of the present paper was to assess people's ability to place self-events and other-events in time. We have previously argued that people use various sources of information to concoct the date of an event (Thompson et al., 1993). Sometimes, they might know the exact date (e.g., a birthday). Sometimes, they might only know the day of the week (e.g., it was a Monday). Sometimes, they might only be able to make a guess at a date on the basis of the details of the events (e.g., the leaves were turning, so it must have been in the autumn). In the present paper, we highlight these knowledge sources by examining the event dates provided by participants in various ways.

Factors related to exact date knowledge. In one set of analyses, we were interested in the factors related to people's recall for exact event dates. For this analysis, we coded event dates as exact or inexact: Because one could estimate an event's exact date, events were scored as exactly dated only if participants both provided a correct date and reported that they knew the exact date. These 
exact recall scores were examined in regressions using our four main predictors (person typicality, event valence, event extremity, and diary type).

The results of these analyses yielded only three statistically significant effects. First, as one might expect, people more often knew the exact date of self-events $(M=.150)$ than of other-events $[M=.092, F(1,4265)=$ $28.93, p<.001]$. Second, people more often knew the exact dates of extreme events than of more moderate events $[F(1,4265)=8.01, p<.01, \beta=.041]$. Finally, people more often knew the exact dates of pleasant events than of unpleasant events $[F(1,4265)=11.39, p<.01$, $\beta=.048]$. Neither the person-typicality effect nor any higher order interactions were statistically reliable.

Relations among event valence, event extremity, diary target, person typicality, and estimation error. We were also interested in knowing what factors are related to estimation error when the exact date of an event is not known. To investigate this issue, we eliminated all events that were both dated exactly and reported as known exactly. For the remaining events, we then calculated the absolute (i.e., unsigned) dating error that participants made for each event. In a series of regression analyses, we then examined the relations between our four main predictors and this estimation error measure.

The results of these analyses revealed that dating error decreased as events became more extreme $[F(1,3731)=$ $6.71, p<.01, \beta=-.044]$ and as events became more person atypical $[F(1,3731)=9.28, p<.01, \beta=.057]$. These outcomes replicate those obtained in Skowronski et al. (1991). The previous study also revealed that positive events were more accurately dated than negative events, and though consistent with this outcome, this ef- fect was not significant in the present study $[F(1,3731)=$ $3.20, p=.07, \beta=-.029$ ].

Examining the role of event memory in event date estimation. People can estimate event dates by using their recall of event details. In general, if event details give clues about event dates, then better event recall should produce lower error in date estimation. Furthermore, if the relations among several of our predictors and dating accuracy are mediated by event memory, then adding the memory measure to the regression models may eliminate the relations between these predictors and dating error. We examined these possibilities by again analyzing estimation error, but in these analyses we included event memory as a covariate.

The results indicate that higher memory ratings were, indeed, strongly associated with lower error in dating estimates $[F(1,3730)=191.18, p<.001, \beta=-.230]$. Furthermore, inclusion of this memory measure in the analyses as a covariate essentially eliminated all other effects. Person-typical events were not significantly associated with larger dating errors than person-atypical events $[F(1,3730)=3.90, p=.05, \beta=.035]$, and extreme events were not significantly associated with lower error than were less extreme events $[F(1,3730)=$ $.80, p>.37]$. Thus, these results indicate that event memory may play an important role in date estimation, both through its direct relation to recall and as a mediator of the relation between various stimulus-based characteristics and recall.

The use of within-week information in the construction of date estimates. Several studies suggest that another type of information used in event dating is within-week information (e.g., Skowronski et al., 1991;

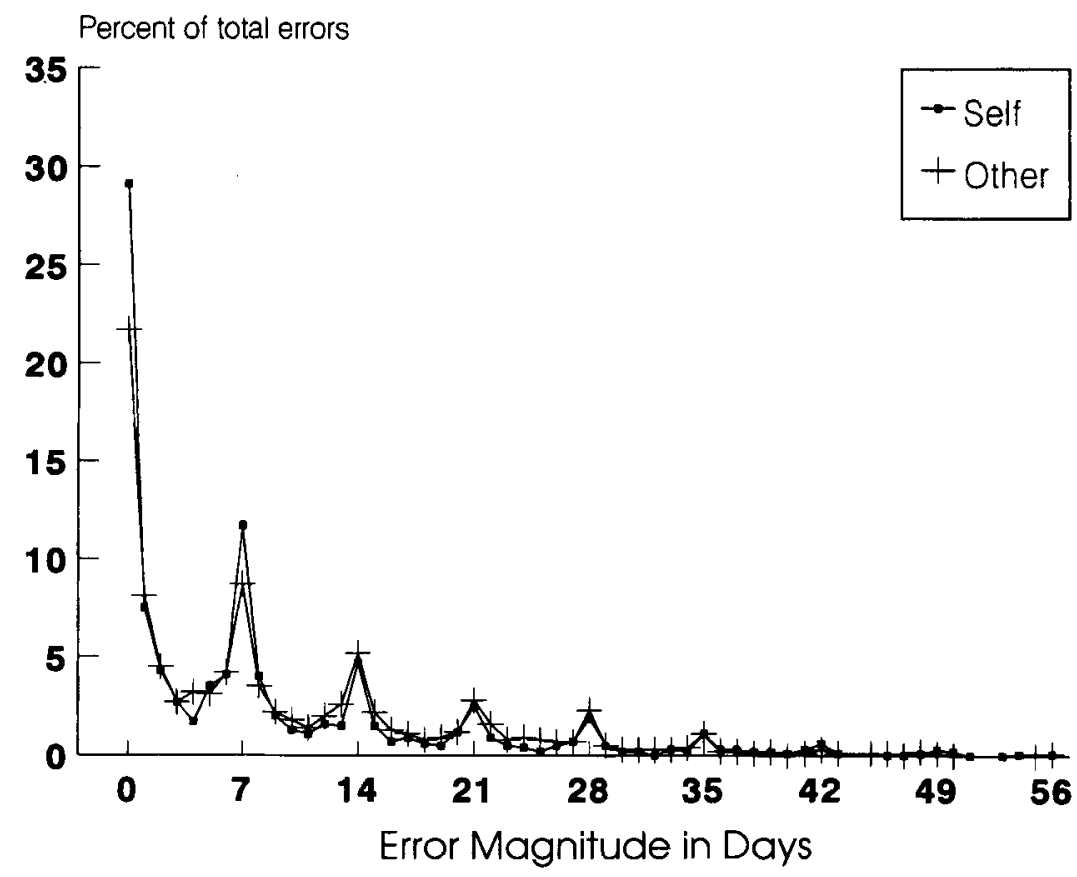

Figure 1. Percent of dating errors as a function of error magnitude and diary target. 
Thompson et al., 1993; see also Huttenlocher, Hedges, \& Prohaska, 1992). For example, in reconstructing an event's date, people may sometimes remember or reconstruct the day of the week on which an event occurred. If people are able to remember or reconstruct the day of the week on which an event occurred, but not the precise date, then it follows that there should be a heightened frequency of errors as a multiple of 7 days. Figure 1 depicts this strong day-of-week pattern in the present data. The absence of substantial self-other differences suggests that many of these day-of-week errors are due to people's use of generic real-world knowledge structures, and not to the use of idiosyncratic information that is directly recalled about events.

Another noteworthy aspect of the data in Figure 1 is the regular scalloping between the day-of-week peaks. We have argued that this regularity may reflect people's use of other recalled or reconstructed within-week information in deriving date estimates (Thompson et al., 1993). For example, the nature of an event (e.g., something that happened at school) might allow one to know that an event was a weekday event and not a weekend event.

One method useful in exploring this possibility is a within-week confusion matrix (Larsen \& Thompson, 1995). This matrix depicts the tendency for individuals to date events incorrectly as a function of day of week: Given an actual day of occurrence, one calculates the likelihood that an event is reported on each day of the week. For example, given that an event occurs on a Monday, one would assess the frequency with which the incorrect dates were placed on another Monday, on a Tuesday, on a Wednesday, and so forth.

We constructed two such $7 \times 7$ confusion matrices (see Table 3), one for self-events and one for other-events. The entries into the matrices presented in Table 3 are derived only from erroneously dated events (i.e., all dating errors of zero days are discarded). By examining the patterns of data across all the days of the week, one can get a sense of the within-week temporal units that participants may be using in date estimation.

Inspection of the data in Table 3 reveals several interesting patterns. First, the elevated values along the main diagonal (upper left to lower right) indicate that, even when exactly dated events are eliminated from the data, people are often still able to reconstruct the correct day of the week on which an event occurred. However, the probability patterns elsewhere in these tables are also quite revealing. For instance, the data indicate that events that occurred on weekdays are relatively unlikely to be placed on weekends, and vice versa. Additionally, the data suggest that for weekdays, people have knowledge of early-week, mid-week, and late-week events (e.g., Monday events are most often erroneously dated on Monday through Wednesday, Tuesday events on Tuesday through Thursday, and so on). The error pattern also suggests that Friday events are more likely to be misdated as Saturday events than as events that occurred on other weekdays. Hence, rather than being work-week events, Friday events seem to be categorized as part of the weekend.

There were also a couple of intriguing asymmetries that we did not expect. Saturday events are relatively likely to be misplaced as occurring on a Sunday, but Sunday events are relatively unlikely to be misplaced as occurring on a Saturday. Similarly, but a bit less obviously, Friday events were more likely to be misdated as occurring on a Saturday than Saturday events were to be misdated as occurring on a Friday. The reasons for these asymmetries are unknown, but they may have to do with the types of events listed. For example, events that were listed for Saturday events may have been "all-purpose" weekend events that could occur on Friday, Saturday, or Sunday (e.g., parties), whereas Sunday events (e.g., church) may have tended to be highly specific to Sunday.

In general, the probabilities in Table 3 are consistent with the notion that people use within-week information to reconstruct event dates. The patterns of errors indicate that two main units of within-week structure are weekends (Friday-Sunday) and the work week (MondayThursday). Furthermore, finer structure may exist within each of those divisions. The work week seems to have early-, mid-, and late-week components, and weekend events also seem to be subcategorized.

One other striking aspect of these data is the high degree of similarity in the errors made for self-events and for other-events. This similarity continues to suggest that these dating errors are not due to specific memories for events, but instead reflect real-world knowledge that applies equally well to either self-events or other-events.

Information source use $\times$ diary type. The high degree of similarity in event dating for self-events and other-events is also apparent from examination of par-

Table 3

Confusion Matrix of Days of the Week for Erroneously Dated Events $\times$ Self-Events and Other-Events

\begin{tabular}{|c|c|c|c|c|c|c|c|c|c|c|c|c|c|c|}
\hline & \multicolumn{7}{|c|}{ Self-Events } & \multicolumn{7}{|c|}{ Other-Events } \\
\hline & Sun & Mon & Tue & Wed & Thu & Fri & Sat & Sun & Mon & Tue & Wed & Thu & Fri & Sat \\
\hline Sun & 38.1 & 6.0 & 3.4 & 4.2 & 2.0 & 2.6 & 6.8 & 26.5 & 7.5 & 3.4 & 3.4 & 5.0 & 5.9 & 10.5 \\
\hline Mon & 6.7 & 22.9 & 17.2 & 12.5 & 8.9 & 5.8 & 3.4 & 7.6 & 16.3 & 15.9 & 13.6 & 10.0 & 10.3 & 5.9 \\
\hline Tue & 4.9 & 16.0 & 24.7 & 14.9 & 16.6 & 8.5 & 5.1 & 5.8 & 20.3 & 20.9 & 19.3 & 13.1 & 10.3 & 3.2 \\
\hline Wed & 8.1 & 25.6 & 21.8 & 31.5 & 26.7 & 14.3 & 7.1 & 12.5 & 23.0 & 19.7 & 25.2 & 21.2 & 14.3 & 10.4 \\
\hline Thu & 5.3 & 14.8 & 22.6 & 19.9 & 27.1 & 12.5 & 9.7 & 13.5 & 9.7 & 19.7 & 20.9 & 24.0 & 11.8 & 3.7 \\
\hline Fri & 9.9 & 9.5 & 8.8 & 10.4 & 11.7 & 37.5 & 24.0 & 9.9 & 14.0 & 12.6 & 12.8 & 17.2 & 34.3 & 25.0 \\
\hline Sat & 26.9 & 5.2 & 1.6 & 6.6 & 6.8 & 18.8 & 43.9 & 24.2 & 9.2 & 8.0 & 4.7 & 9.5 & 13.2 & 41.4 \\
\hline
\end{tabular}

Note-Columns are actual days, rows are reported days, and entries are conditional probabilities. 
ticipants' self-reports of the sources of information used to date events. Recall that, after they had provided each event date, participants were asked to select one of eight sources of information that they had used to construct the event date. Table 4 presents the proportions of exact dating and dating error magnitude for each information source for both self-events and other-events.

The data in Table 4 suggest that information source use for self-events and other-events differed substantially in only two categories: exact date known (used more often for self-events) and prototypic information (used more often for other-events). These data also show that selfother differences in dating accuracy are rather minimal when self-events and other-events are dated using the same information source. Hence, self--other differences in event dating may be partially due to information sources that are differentially available; when the information sources are similar, the associated dating performance is also quite similar.

The effects of boundaries on dating error. Boundaries can also affect event dating performance. Research shows that the boundary dates of diary studies can produce systematic errors in the event dates that participants provide (see, e.g., Thompson et al., 1993). Events that are relatively old (e.g., near the start date of the study)

Table 4

Participants' Reports of Information Use in Event Dating by Dating Strategy and Diary Target: Percentage Used, Error Magnitude, and Exact Dating Accuracy

\begin{tabular}{|c|c|c|}
\hline \multirow[b]{2}{*}{ Strategy } & \multicolumn{2}{|c|}{ Diary Event Target } \\
\hline & Self & Other \\
\hline \multicolumn{3}{|l|}{ Exact date known } \\
\hline$\%$ used & 19.02 & 11.80 \\
\hline Error magnitude & 1.93 & 2.70 \\
\hline$\%$ exactly correct & 79.63 & 79.15 \\
\hline \multicolumn{3}{|l|}{ Used reference event } \\
\hline$\%$ used & 9.83 & 10.79 \\
\hline Error magnitude & 7.40 & 6.72 \\
\hline$\%$ exactly correct & 31.86 & 33.95 \\
\hline \multicolumn{3}{|c|}{ Knew general reference period } \\
\hline$\%$ used & 45.87 & 40.76 \\
\hline Error magnitude & 8.66 & 9.25 \\
\hline$\%$ exactly correct & 18.03 & 14.16 \\
\hline \multicolumn{3}{|c|}{ Estimated no. intervening events } \\
\hline$\%$ used & 4.74 & 5.17 \\
\hline Error magnitude & 12.14 & 11.76 \\
\hline$\%$ exactly correct & 7.34 & 7.77 \\
\hline \multicolumn{3}{|c|}{ Used memory clarity to estimate } \\
\hline$\%$ used & 4.44 & 4.62 \\
\hline Error magnitude & 11.46 & 13.94 \\
\hline$\%$ exactly correct & 24.51 & 18.48 \\
\hline \multicolumn{3}{|c|}{ Used prototypic information } \\
\hline$\%$ used & 15.49 & 25.60 \\
\hline Error magnitude & 13.76 & 14.13 \\
\hline$\%$ exactly correct & 7.30 & 5.69 \\
\hline \multicolumn{3}{|l|}{ Guess } \\
\hline$\%$ used & .61 & 1.20 \\
\hline Error magnitude & 15.93 & 8.54 \\
\hline$\%$ exactly correct & 14.29 & 25.00 \\
\hline \multicolumn{3}{|l|}{ Other } \\
\hline$\%$ used & .00 & .05 \\
\hline Error magnitude & - & 6.00 \\
\hline$\%$ exactly correct & - & 0.00 \\
\hline
\end{tabular}

evince telescoping: They are dated as more recent than they actually are. Events that are relatively young (e.g., events that occurred near the diary test) evince time expansion: They are dated as older than they actually are. As noted elsewhere (see Rubin \& Baddeley, 1989), one explanation for this pattern of results is that knowledge of the exact dates provided by the boundary events limits the direction of the dating errors that can be made in the estimation of dates of nonboundary events. Thus, dating errors ought to show evidence of regression to the mean of the boundary period, but because the magnitude of the errors is greater for older events than for younger events, the telescoping error for older events ought to be bigger than the time expansion error for younger events.

We attempted to duplicate this finding, and furthermore, we investigated whether these effects were equally strong for self-events and for other-events. Because date estimates for other-events have errors of greater magnitude than those for self-events, we expected that these boundary effects might be greater for the other-events. To investigate this possibility, we analyzed the raw (signed) dating error using our pooled within-subject regression technique.

Figure 2 depicts the 5-day running average for the dating errors produced for self-events and other-events (a running average was used to make the effects more easily viewed). As illustrated in Figure 2, the results replicate the basic boundary effects, showing both telescoping for events near the start of the study and time expansion for events near the diary test date. In terms of the regression analyses, both the linear effect of event age $[F(1,4268)=798.16, p<.0001, \beta=-.389]$ and the quadratic effect of event age $[F(1,4268)=99.00$, $p<.0001, \beta=-.150$ ] significantly predicted dating error. As expected, we also found that the magnitude of these boundary effects depended on the diary type: In particular, telescoping effects were greater for otherevents than for self-events. Statistically, this effect is revealed in the interaction between diary target and the linear component of event age $[F(1,4267)=16.98, p<$ $.0001]$.

\section{DISCUSSION}

This study employed a diary methodology to investigate recall for autobiographical events and for the dates that people assign to these events. Furthermore, this study attempted to gather information concerning some of the underlying cognitive processes involved in event memory and event dating. For event memory, we assessed how initial mental involvement and event rehearsal affected self-event and other-event recall, as well as whether these variables mediated other effects. For event dating, we conducted several analyses designed to explore the multiple sources of information that people access in the process of reconstructing an event date. Finally, for both memory and dating, we were explicitly concerned with a comparison of self-events to otherevents, with a particular eye toward data suggesting sig- 


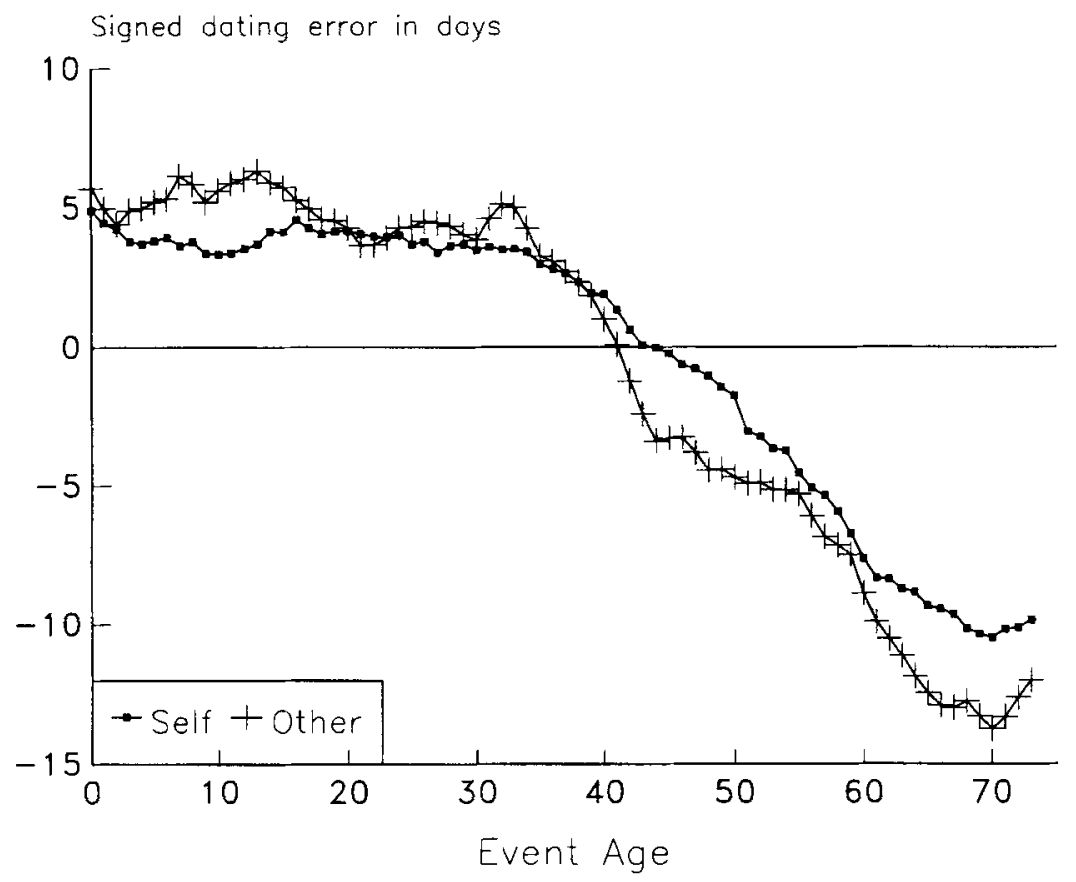

Figure 2. Signed dating error (running 5-day average) as a function of retention interval for self-events and other-events.

nificant differences in the processing of self-information and other-information.

Some of the outcomes that we obtained in our investigation of event memory were straightforward. Self-events were recalled better than other-events. Extreme events were recalled better than less extreme events. Events that were rehearsed frequently were recalled better than events that were rehearsed less frequently. Recall for person-atypical information was better than recall for person-typical or person-neutral events.

Some of the results that we obtained might be, to some, surprising. One such result is the reversal of the positivity bias obtained in recall for other-events. That is, the data from both this study and our earlier study (Skowronski et al., 1991) suggest that, other things being equal, a person has better recall for positive self-events, but better recall for negative other-events. This work conceptually replicates laboratory work by Skowronski and Carlston (1987), who found that people tended to recall negative information about others better than positive information (also see Bird, 1987; Pratto \& John, 1991). Such an outcome makes sense from the cognitive processing perspective outlined by Fiske (1980), who proposed that negative events are particularly attention drawing. Thus, if heightened attention leads to recall, it makes sense that others' negative events, which garner heightened attention, would elicit heightened recall. However, the rehearsal data suggest that attention is not the only viable explanation for the negativity effect in other-event recall. Our participants reported that they rehearsed others' negative events more than they rehearsed others' positive events; our analyses suggest that this dif- ferential rehearsal can account for the negativity bias in other-event recall.

By comparison, participants also reported that they recalled their own positive events better than their own negative events. This positivity effect was no surprise. However, surprising indeed was that, in contrast to those who believe that people repress the negative when they think about the past, participants in our study reported that they actually rehearsed negative self-events more frequently than positive self-events. As a result, differential rehearsal cannot account for positivity in selfevent recall. Instead, the data suggest that initial mental involvement may play a significant role in the enhanced recall of positive self-events. Participants reported more initial processing of positive self-events than negative self-events, and regression analyses confirmed that initial mental involvement is a plausible mediator of this positivity effect.

Given past research suggesting that people are optimistic, accentuate the positive, and remember the past through rose-colored glasses (e.g., Matlin \& Stang, 1978), the finding that participants report more frequent rehearsal for negative events than for positive events is intriguing. However, it is always possible that this rehearsal difference does not actually exist. Because rehearsals were reported retrospectively, people may have simply selectively forgotten their rehearsals of positive events, causing an overestimation of negative event rehearsals relative to positive event rehearsals (for a similar suggestion, see Rubin \& Kozin, 1984). Alternatively, the relatively high rehearsal accorded to negative events may occur because of salience effects. That is, if recall 
of pleasant events is the norm, then recall of aversive events stands out by comparison. Hence, because one can more easily recall specific instances in which negative events were rehearsed, negative event rehearsal rates may be inflated. The data in the present study do not discount these possibilities.

Furthermore, even if people do rehearse negative events more frequently than positive events, the possibility remains that people rehearse negative self-events and other-events for different reasons. Negative selfevents may be rehearsed and analyzed to improve one's life or to avoid unhappy circumstances that might again arise without precaution. By comparison, negative otherevents might be rehearsed to gain a sense of predictability regarding another's behavior. These issues remain to be explored by future research.

Nonetheless, our data suggest danger in focusing solely on an event's emotional tone, without consideration of other factors potentially involved. For example, Peeters and Czapinski (1990) have proposed that people will avoid negative information because it is threatening. This implies a high level of negative event forgetting, regardless of whether the negative events are self-events or other-events. Obviously, our data are not congruent with this idea. Similarly, Taylor (1991) proposed that initial mobilization of responses (cognitive and otherwise) to negativity should be strong, but that negativity effects should dampen over time. This perspective also has difficulty accounting for the differing impact of event valence on self-event and other-event recall observed in this study. ${ }^{5}$

The profound self-other difference in the relation between valence and recall stands in stark contrast to the overall absence of such differences in event dating. Although self-dating tended to be more accurate than other-dating, the patterns that emerged for self-events and for other-events were otherwise highly similar. Generally, no important interactions between the diary type variable and other predictors in the regressions emerged. In general, people were more likely to recall the exact dates of self-events, extreme events, and pleasant events. When people could not recall the exact dates of events, date estimates showed less error if the events were selfevents, extreme, and atypical. However, an interaction with diary target did emerge in the boundary analyses. The results of dating boundary analyses were consistent with previous research in that both time expansion and telescoping effects emerged. However, the data also indicated that both time expansion errors and especially telescoping errors were greater for other-events than for self-events.

We also explored some information sources that people use to construct their date estimates. One information source is direct date recall. Analyses indicated that recall of the exact date of the event was better (1) for positive than for negative events, (2) for extreme than for moderate events, and (3) for self-events than for otherevents. When exact dates cannot be recalled, those dates must be reconstructed, and a primary source of recon- struction is event memory. Our analyses suggest that increases in memory are negatively related to estimation error. These analyses also suggest that event memory may serve as a mediator between estimation error and the predictors of event extremity and person typicality.

Other information sources, aside from date recall and event memory, may be used in the construction of an event date. Two additional sets of analyses suggest that within-week information contributes to event date reconstruction. The first analysis documents that a high proportion of errors are likely to be day-of-week errors: right day of week, but wrong exact date. The second analysis suggests that within-week estimation errors follow somewhat broader patterns. Specifically, participants have a fair amount of knowledge about whether an event was a weekend (Fri-Sun) or weekday (Mon-Thurs) event and also have some idea of whether the event occurred early or late in these two time periods. Again, there were no substantial self-other differences in these patterns. The absence of self-other differences suggests that participants were sometimes using generic knowledge about the world (e.g., parties are generally on Friday or Saturday, church on Sunday), in addition to their event-specific knowledge, to construct their estimates.

We also asked participants to report directly the information sources that they used to date the events. The pattern of source usage was quite similar for self-events and other-events. Furthermore, some types of information led to more accurate date estimates than did other sources. These effects were generally sensible, in that sources that should lead to high accuracy (e.g., exact date known, relating events to other (dated) events) generally did so, whereas strategies that ought to be inherently less accurate (e.g., memory clarity, guessing) generally produced correspondingly inaccurate date estimates.

The self-report methods used in this study raise several issues. To the extent that participants do not have insight into their own cognitive processes, they may not be able to report on those processes accurately. Consequently, the self-reports might be biased. For example, consider our memory measure, essentially a self-report of the "goodness" of a memory. These ratings are probably not perfect, and (rightly) skeptical readers are likely to point to potential biases in this measure to explain our findings. For example, we may have obtained extremity effects in recall because people think that extreme events are better recalled, so they respond accordingly. Similarly, the impact of event valence on recall may represent mere expectations about the relationship between event valence and recall.

We firmly believe that these issues should be kept in mind in regard to our results. However, by the same token, these concerns should not be overstated. For example, the data in Table 4 clearly indicate a strong relation between self-reported information source and dating accuracy. Participants often knew when their date estimates were exact, or were close to being correct, and dating accuracy was obviously dependent on the information source being used. This suggests that partici- 
pants' perceptions of dating accuracy map relatively well on to their actual dating performance-when they thought their event date was exact, they were right about $80 \%$ of the time. Similarly, people's event memory ratings strongly predicted dating error, a relation that is most sensibly explained by the fact that the reconstruction of an event date often depends on the details of memory. Thus, these data indicate that people apparently $d o$ often have access to their cognitive processes and can report on them with reasonable accuracy.

However, it would be desirable for future research to bring alternative techniques to bear on the issue of autobiographical self-event and other-event recall and event dating and, more importantly, to use techniques that do not depend so heavily on self-report assessments of memory. This seems possible. For example, Fuhrman and Wyer (1988) employed a response-time paradigm to assess individuals' performance in a temporal order judgment task (e.g., "Which event occurred sooner?"). From those data, Fuhrman and Wyer concluded that the way in which individuals temporally categorized events affected their performance. However, Fuhrman and Wyer selected only a very small sample of autobiographical events for their study. We can conceive of a diary study that collects relatively large amounts of autobiographical events, exploring how various factors (valence, self- versus other-events) affect temporal judgment performance. Other studies employing more usual measures of memory are also real possibilities.

\section{REFERENCES}

BIRD, C. P. (1987). The influence of the spacing of trait information on impressions of likability. Journal of Experimental Social Psychology, 23, 481-497.

BREWER, W. F. (1988). Memory for randomly sampled autobiographical events. In U. Neisser \& E. Winograd (Eds.), Remembering reconsidered: Ecological \& traditional approaches to the study of memory (pp. 21 -90). Cambridge: Cambridge University Press.

Brown, N. R. (1990). Organization of public events in long-term memory. Journal of Experimental Psychology: General, 119, 297-314.

Brown, R., \& Kulik, J. (1977). Flashbulb memories. Cognition, 5, 73-99.

BURT, C. D. B., \& KEMP, S. (1991). Retrospective duration estimates of public events. Memory \& Cognition, 19, 252-262.

COHEN, G. (1989). Memory in the real world. Hove, U.K.: Erlbaum.

COHEN, J., \& COHEN, P. (1983). Applied multiple regression/correlation analysis for the behavioral sciences ( 2 nd ed.). Hillsdale, $\mathrm{NJ}$ : Erlbaum.

CONWAy, M. A. (1990). Autobiographical memory: An introduction. Philadelphia: Open University Press.

Diener, E., LARSEN, R. J., LeVine, S., \& Emmons, R. A. (1985). Intensity and frequency: Dimensions underlying positive and negative affect. Journal of Personality \& Social Psychology, 48, 1253-1265.

FISKE, S. (1980). Attention and weight in person perception: The impact of negative and extreme behavior. Journal of Personality \& Social Psychology, 38, 889-906.

Freud, S. (1957). Repression. In J. Strachey (Ed and Trans.), The standard edition of the complete psychological works of Sigmund Freud (Vol. 19, pp. 146-158). London: Hogarth Press. (Original work published 1915)

Friedman, W. J. (1993). Memory for the time of past events. Psychological Bulletin, 113, 44-66.
Fuhrman, R. W., \& WYer, R. S., JR. (1988). Event memory: Temporalorder judgments of personal life experiences. Journal of Personality \& Social Psychology, 54, 365-384.

HASTIE, R. (1984). Causes and effects of person attribution. Journal of Personality \& Social Psychology, 46, 44-56.

HerrmanN, D. J. (1984). Questionnaires about memory. In J. E. Harris \& P. E. Morris (Eds.), Everyday memory, actions \& absent-mindedness (pp. 133-151). New York: Academic Press.

HerrmanN, D. J., \& NeIsSER, U. (1978). An inventory of everyday experiences. In M. M. Gruneberg, P. E. Morris, \& N. Sykes (Eds.), Practical aspects of memory (pp. 35-51). San Diego: Academic Press.

Huttenlocher, J., Hedges, L., \& Bradburn, N. (1990). Reports of elapsed time: Bounding and rounding processes in estimation. Journal of Experimental Psychology: Learning, Memory, \& Cognition, 16, 196-213.

Huttenlocher, J., Hedges, L., \& Prohaska, V. (1992). Memory for day of the week: A $5+2$ day cycle. Journal of Experimental Psychology: General, 121, 313-325.

Kreitler, H., \& Kreitler, S. (1968). Unhappy memories of "the happy past": Studies in cognitive dissonance. British Journal of Psychology, 59, 157-166.

LARSEN, S. F. (1988). Remembering without experiencing: Memory for reported events. In U. Neisser \& E. Winograd (Eds.), Remembering reconsidered: Ecological \& traditional approaches to the study of memory (pp. 326-355). New York: Cambridge University Press.

LARSEN, S. F., \& THOMPSON, C. P. (1995). Reconstructive memory in the dating of personal and public news events. Memory \& Cognition, 23, 780-790.

LeONesio, R. J., \& Nelson, T. O. (1990). Do different metamemory judgments tap the same underlying aspects of memory? Journal of Experimental Psychology: Learning, Memory, \& Cognition, 16, 464-470.

LinTon, M. (1975). Memory for real-world events. In D. A. Norman \& D. E. Rumelhart (Eds.), Information processing: Explorations in cognition (pp. 376-404). San Francisco: Freeman.

Matlin, M. W., \& Stang D. J. (1978). The Pollyanna principle. Cambridge, MA: Schenkman.

NEISSER, U. (1982). Memory: What are the important questions? In U. Neisser (Ed.), Remembering in natural contexts (pp. 3-19). San Francisco: Freeman.

Peeters, G., \& Czapinski, J. (1990). Positive-negative asymmetry in evaluations: The distinction between affective and informational negativity effects. European Review of Social Psychology, 1, 33-60.

Pillemer, D. B. (1984). Flashbulb memories of the assassination attempt on President Reagan. Cognition, 16, 63-80.

Pillemer, D. B., Goldsmith, L. R., Panter, A. T., \& White, S. H. (1988). Journal of Experimental Psychology: Learning, Memory, \& Cognition, 14, 709-715.

PRatTo, F., \& JOHN, O. P. (1991). Automatic vigilance: The attentiongrabbing power of negative social information. Journal of Personality \& Social Psychology, 61, 380-391.

Reisberg, D., Heuer, F., MCLean, J., \& O'Shaughnessy, M. (1988). The quantity, not the quality, of affect predicts memory vividness. Bulletin of the Psychonomic Society, 26, 100-103.

RoBinson, J. A. (1992). First experience memories: Contexts and functions in personal histories. In M. A. Conway, D. C. Rubin, H. Spinnler, \& W. A. Wagenaar (Eds.), Theoretical perspectives on autobiographical memory (pp. 223-240). Dordrecht: Kluwer

RuBIN, D. C. (1982). On the retention function for autobiographical memory. Journal of Verbal Learning \& Verbal Behavior, 21, 21-38.

RuBIN, D. C. (ED.) (1996). Remembering our past: Studies in autobiographical memory. New York: Cambridge University Press.

RuBIN, D. C., \& BADDELEY, A. D. (1989). Telescoping is not time compression: A model of the dating of autobiographical events. Memory \& Cognition, 17, 653-661

Rubin, D. C., \& Kozin, M. (1984). Vivid memories. Cognition, 16, 81-95.

Skowronski, J. J., Betz, A. L., Thompson, C. P., \& Larsen, S. (1995) Long-term performance in autobiographical event dating: Patterns of accuracy and error across a two-and-a-half year time span. In A. F. 
Healy \& L. E. Bourne (Eds.), Learning and memory of knowledge and skills (pp. 206-233). Hillsdale, NJ: Erlbaum.

Skowronski, J. J., Betz, A. L., Thompson, C. P., \& Shannon, L. (1991). Social memory in everyday life: Recall of self-events and other-events. Journal of Personality \& Social Psychology, 60, 831-843.

Skowronski, J. J., \& CarLston, D. E. (1987). Social judgment and social memory: The role of cue diagnosticity in negativity, positivity, and extremity biases. Journal of Personality \& Social Psychology, 52, 689-699.

Srull, T. K., Lichtenstein, M., \& Rothbart, M. (1985). Associative storage and retrieval processes in person memory. Journal of Experimental Psychology: Learning, Memory, \& Cognition, 11, 316-345.

STANGOR, C., \& MCMillan, D. (1992). Memory for expectancycongruent and expectancy-incongruent information: A review of the social and social-developmental literatures. Psychological Bulletin, $111,42-61$.

TAYLOR, S. E. (1991). Asymmetrical effects of positive and negative events: The mobilization-minimization hypothesis. Psychological Bulletin, 110, 67-85

ThOMAs, D. L., \& DiENER, E. (1990). Memory accuracy in the recall of emotions. Journal of Personality \& Social Psychology, 59, 291-297.

Thompson, C. P. (1982). Memory for unique personal events: The roommate study. Memory \& Cognition, 10, 324-332.

Thompson, C. P. (1985). Memory for unique personal events: Effects of pleasantness. Motivation \& Emotion, 9, 277-289.

Thompson, C. P., Skowronski, J. J., \& BETZ, A. L. (1993). The use of partial temporal information in dating personal events. Memory \& Cognition, 21, 352-360.

Thompson, C. P., Skowronski, J. J., LARSEN, S., \& Betz, A. L. (1996). Autobiographical memory: Remembering what and remembering when. Mahwah, NJ: Erlbaum.

Thompson, C. P., Skowronski, J. J., \& LeE, D. J. (1988). Telescoping in dating naturally-occurring events. Memory \& Cognition, 16, 461-468.

WAGENAAR, W. (1986). My memory: A study of autobiographical memory over 6 years. Cognitive Psychology, 18, 225-252.

WinOGRAD, E., \& KILLINGER, W. A., JR. (1983). Relating age at encoding in early childhood to adult recall: Development of flashbulb memories. Journal of Experimental Psychology: General, 112, 413-422.

\section{NOTES}

1. Although this measure of extremity is indirect because it is derived from another measure, other research (Skowronski et al., 1995) revealed no differences between direct and indirect measures of extremity.

2. In almost all cases, in the present paper we report raw means rather than adjusted (least squares) means. The simple reason for this is that the adjustments made in the course of running the regressions did not substantially affect the relation between or among significantly different means. To avoid redundancy, however, we report adjusted means in the covariance analyses.

3. One possible concern about the memory and dating error results that we report in this paper is that these results might be due to differences in event content. For example, extreme events may pertain more to the family, whereas moderate events may pertain more to the workplace. If event memory or dating error is greater for family events than workplace events, the apparent extremity effects that we obtained would be illusory. The same logic could also extend to the other significant effects we obtained. Using a content-coding scheme originally described in Skowronski et al. (1991), we examined this possible content confound. This hierarchical coding scheme classifies events into 73 separate categories, such as events involving mothers, events involving fathers, events at church, events at school, sports-related events, and so on. The entry of an event content term into the regression models does not affect our basic results for either memory or dating error; hence, our results are not likely due to event content differences.

4. Two additional effects that were not significant in the original analyses were significant in these new analyses. These were a three-way interaction among event valence, event extremity, and person typicality $[F(1,4254)=10.30, p<.001]$ and an event extremity $\times$ diary target interaction $[F(1,4258)=8.30, p<.01]$. In contrast to the positivity effect in self-event recall, which we obtained in our earlier study, we had no reason to expect these effects to occur (i.e., they did not emerge in our earlier research), so we omit extended discussion of them.

5. However, in fairness to the Taylor (1991) approach, there is room in her proposal for negativity to mobilize different resources in the case of self-events and other-events, and these differences may account for the data obtained here.

(Manuscript received June 21, 1995;

revision accepted for publication July 15, 1996). 